\title{
Distorção da imagem corporal em adolescentes: um estudo de comparação entre dois instrumentos
}

\author{
Adolescents' body image distortion: a comparison study \\ between two instruments
}

Maria Fernanda Laus ${ }^{1}$, Telma M. Braga Costa², Sebastião S. Almeida ${ }^{3}$

\begin{abstract}
RESUMO
Objetivo: comparar dois instrumentos classicamente utilizados pela literatura especializada, indicados para avaliar possíveis distorções da imagem corporal em adolescentes e adultos de ambos os sexos. Metodologia: aplicou-se o Questionário de Imagem Corporal (BSQ) e a Escala de Figuras de Silhuetas (EFS) em 118 estudantes, com idade média de 16,5 anos $( \pm 1,2)$, sendo 62 meninos e 56 meninas, de 4 escolas públicas estaduais. O primeiro instrumento mede as preocupações com a forma do corpo, a auto-depreciação devido à aparência física e a sensação de estar "gordo"; enquanto o segundo avalia a percepção do estado atual e do estado desejado, através de 15 silhuetas de cada gênero, apresentadas em cartões individuais, com variações progressivas na escala de medidas, da figura mais magra $\left(\mathrm{IMC}=12,5 \mathrm{Kg} / \mathrm{m}^{2}\right)$ para a mais larga $\left(\mathrm{IMC}=47,5 \mathrm{Kg} / \mathrm{m}^{2}\right)$. Além disso, aferiu-se peso e altura para cálculo do Índice de Massa Corporal (IMC) e posterior comparação com a EFS. A análise dos resultados foi realizada através da escala de avaliação do BSQ e o tratamento estatístico consistiu na análise descritiva dos resultados, além da aplicação de um teste de correlação de Pearson entre os instrumentos e do Teste $t$ de Student para comparação entre os sexos. Resultados: através do BSQ observou-se que $14,5 \%$ dos meninos e $60,7 \%$ das meninas apresentaram algum grau de distorção da imagem corporal. Já a EFS demonstrou que no grupo feminino, o IMC médio escolhido como "atual" foi de $26,9 \mathrm{Kg} / \mathrm{m}^{2}$, enquanto o IMC médio real foi de $21,5 \mathrm{Kg} / \mathrm{m}^{2}$ e no grupo masculino, o IMC médio "atual" escolhido foi $23,7 \mathrm{Kg} / \mathrm{m}^{2}$, enquanto a média do IMC real foi de $21,0 \mathrm{Kg} / \mathrm{m}^{2}$, indicando uma prevalência de distorção da imagem corporal em ambos os sexos. A análise estatística mostrou uma correlação positiva significativa $(r=0,38)$ entre os dois instrumentos, entretanto, a EFS não apresentou diferença devido ao sexo $(p=0,93)$, enquanto o BSQ detectou um maior grau de distorção no sexo feminino $[F(1,110)=13,80$; $p<0,001)]$. Conclusão: ambos os instrumentos detectaram distorções da imagem corporal, porém, o BSQ conseguiu detectar diferenças devido ao sexo enquanto a EFS parece não ser suficientemente sensível para diferenciar os dois sexos.
\end{abstract}

Palavras-chave: Adolescente. Imagem Corporal. Distorção da Percepção. Métodos de Avaliação.

1. Nutricionista. Pós-graduanda - Departamento de Psicologia e Educação da Faculdade de Filosofia, Ciências e Letras de Ribeirão Preto - USP (FFCLRP-USP)

2. Docente. Coordenadora do Curso de Nutrição da Universidade de Ribeirão Preto (UNAERP)

3. Docente. Departamento de Psicologia e Educação da FFCLRP/ USP
Correspondência:

Maria Fernanda Laus

Laboratório de Nutrição e Comportamento Departamento de Psicologia e Educação - FFCLRP/USP. Av. Bandeirantes, 3900. Monte Alegre 14040-901. Ribeirão Preto - SP. e-mail: fernandalaus@yahoo.com.br

Artigo recebido em 23/07/2008 Aprovado em 30/06/2009 


\section{Introdução}

A imagem corporal tem sido descrita como a capacidade de representação mental do próprio corpo pertinente a cada indivíduo, sendo que esta imagem envolve aspectos relacionados à estrutura (como tamanho, dimensões) e à aparência (forma, aspecto), entre vários outros componentes psicológicos e físicos da imagem corporal. ${ }^{1}$

Estudos realizados em diferentes países nos diversos estágios de desenvolvimento sugerem que grupos jovens apresentam com frequência: insatisfação com a imagem corporal, imagem negativa, temor à obesidade e tendência a utilizarem diferentes técnicas para controle de peso. ${ }^{2,3}$

Dessa forma, torna-se cada vez mais importante o desenvolvimento de instrumentos capazes de detectar possíveis distorções da imagem corporal nos diferentes grupos populacionais, e a escolha adequada deste instrumento é imprescindível para a validade dos resultados.

Os instrumentos mais citados atualmente na literatura para avaliação de distorção da imagem corporal em adolescentes são: o Questionário sobre a Imagem Corporal (BSQ) e a Escala de Figuras de Silhuetas (EFS).

O BSQ foi inicialmente proposto por Cooper et $\mathrm{al}^{4}$ para avaliar distorção da imagem corporal em mulheres com transtornos alimentares (TA), entretanto, já existem estudos que comprovam sua adequada utilização na mensuração da preocupação com a forma corporal e com o peso, especialmente a frequência com que indivíduos de ambos os sexos, com e sem TA, experimentam a sensação de se "sentirem gordos"4. Ele fornece uma avaliação dos distúrbios da imagem corporal tanto em população clínica quanto em população não clínica e pode ser utilizado para avaliar o papel deste distúrbio no desenvolvimento, na manutenção e na resposta ao tratamento de transtornos alimentares. ${ }^{5}$

Um estudo conduzido por Rosen et $\mathrm{al}^{6}$ avaliou as propriedades psicométricas do instrumento em 466 indivíduos de ambos os sexos e encontrou, no testereteste, um coeficiente de fidedignidade $r=0,88$ $(\mathrm{p}<0,001)$ e uma forte correlação com outros instrumentos de avaliação da imagem corporal, concluindo que o BSQ é uma medida válida e confiável deste constructo.

A primeira tradução do BSQ para o português foi realizada por Cordás e Castilho ${ }^{7}$ com aplicação destinada ao sexo feminino. Em 2001 Di Pietro ${ }^{8}$ testou sua validade interna, dimensionalidade e desempenho em uma população de 164 estudantes universitários e o questionário demonstrou uma adequada consistência interna (alfa de Cronbach $=0,96$ ) e manteve as características da escala original.

Outros estudos também têm sido realizados para avaliar a utilização do BSQ em diferentes populações sem TA, e todos têm encontrado bons índices de validade discriminante e concorrente e boa confiabilidade teste-reteste, bem como adequada consistência interna ${ }^{4,6}$.

Já a EFS constitui um instrumento bastante eficaz para avaliar o grau de distorção e insatisfação com o peso e as dimensões corporais, como largamente aceito e aplicado por outros pesquisadores ${ }^{9,10,11}$ sendo válida para estudos quantitativos da percepção da imagem corporal em ambos os sexos. ${ }^{12}$ Inicialmente ela foi desenvolvida por Stunkard et $\mathrm{al}^{13}$ e posteriormente, Thompson e Gray ${ }^{14}$ desenvolveram uma nova versão que consistia em 9 silhuetas de cada gênero e a administração do teste-reteste demonstrou uma fidedignidade $r=0,78(\mathrm{p}<0,05)$ e uma validade de $98 \%$. O estudo de validação da versão brasileira da escala foi realizado por Kakeshita e Almeida ${ }^{15}$ e demonstrou alta fidedignidade no teste-reteste obtida com as silhuetas com valores de 0,87 para o coeficiente de correlação (IC 95\%: 0,79-0,91), indicando que as medidas se mantêm no reteste realizado mesmo depois de decorrido um mês do teste. Estes resultados comprovam a validade interna do instrumento, uma vez que o controle do procedimento empregado resultou em dados pouco variáveis e, portanto, confiáveis e fidedignos. A versão da escala adaptada para o Brasil manteve as características da escala original desenvolvida para o estudo de sujeitos de outras nacionalidades, culturas, idades e nível socioeconômico e a manutenção das características originais exprime razoável segurança na generalização dos achados para outras amostras, conferindo validade externa ao instrumento ${ }^{15}$.

Segundo Leonhard e Barry ${ }^{16}$ os estudos mais antigos relacionados à imagem corporal focavam-se em indivíduos obesos. Mais recentemente, com o aumento da incidência de anorexia e bulimia nervosas, as pesquisas se focaram na imagem corporal desta população. Entretanto, nos últimos anos, distúrbios de imagem corporal têm sido encontrados também em populações eutróficas, sem transtornos alimentares e cada vez mais jovens e vários estudos já foram conduzidos utilizando os instrumentos em questão neste tipo de população. 
Para Thompson et al ${ }^{17}$, a escolha do instrumento a ser utilizado em cada situação específica deve se pautar nos índices psicométricos dessas escalas, assim como em sua adequação à amostra a ser estudada, na medida em que todas elas se assemelham, no que diz respeito a seus pressupostos teóricos.

Uma vez que não há estudos na literatura comparando diferentes instrumentos em adolescentes, o objetivo do presente estudo foi verificar se os dois instrumentos mais utilizados na avaliação de distorções da percepção da imagem corporal (BSQ e EFS) podem indicar diferenças de gênero nesta população.

\section{Metodologia}

\section{Casuística}

Primeiramente o Projeto foi submetido ao Comitê de Ética da Faculdade de Filosofia, Ciências e Letras de Ribeirão Preto - USP e após a aprovação foram entrevistados 118 adolescentes matriculados no ensino médio, de ambos os sexos, com idade média de 16,5 anos $( \pm 1,2)$, sendo 62 meninos e 56 meninas, selecionados aleatoriamente para participar como voluntários do estudo, de quatro escolas públicas escolhidas através de sorteio, situadas nas regiões norte, sul, leste e oeste da cidade de Ribeirão Preto, SP. Como alunos de escolas públicas, a condição socioeconômica dos adolescentes caracteriza uma amostra de baixo poder aquisitivo. Adolescentes com transtornos alimentares ou com algum grau de deficiência física foram excluídos do estudo, uma vez estes podem ter a percepção da imagem corporal alterada em função de sua situação. Não houve recusa por nenhum participante durante a coleta dos dados.

\section{Instrumentos}

\section{Índice de Massa Corporal (IMC)}

É uma medida que expressa a relação entre o peso do sujeito em quilos dividido pela altura em metros ao quadrado ( $\mathrm{IMC}=\mathrm{Kg} / \mathrm{m}^{2}$ ), preconizado pela Organização Mundial de Saúde. ${ }^{18}$ O IMC é um recomendável índice de avaliação do peso corporal, uma vez que tem mostrado uma boa correlação com medidas mais precisas realizadas em laboratório sobre tecido adiposo, além de ser um bom indicador do estado nutricional de um indivíduo. ${ }^{19} \mathrm{~A}$ análise foi realizada de acordo com a classificação preconizada pelo National Center for Chronic Disease Prevention and Health
Promotion. ${ }^{20}$ Para tal, os dados de peso e altura foram colocados no programa EpiInfo, que classificou cada indivíduo de acordo com os limites de Percentis sendo Baixo Peso $\mathrm{P}<5$; Eutrofia $\mathrm{P} 5-\mathrm{P}<85$; Sobrepeso $\mathrm{P} \geq 85-\mathrm{P}<95$ e Obesidade $\mathrm{P} \geq 95$.

\section{Questionário sobre a Imagem Corporal (BSQ)}

Mede as preocupações com a forma do corpo, auto-depreciação devido à aparência física e a sensação de estar "gordo". ${ }^{12}$ Cada questão é composta por seis alternativas de resposta, que variam do "sempre" ao "nunca". Para cada alternativa escolhida são conferidos pontos que variam de 1 a 6 (sempre $=6$; muito frequentemente $=5$; frequentemente $=4$; às vezes $=$ 3 ; raramente $=2 ;$ nunca $=1$ ). O resultado do teste é a somatória dos 34 itens contidos no questionário, e a classificação dos resultados reflete os níveis de preocupação com a imagem corporal. Obtendo resultado menor que 70 pontos, é constatado um padrão de normalidade e tido como ausência de distorção da imagem corporal. Resultados entre 70 e 90 pontos são classificados como leve distorção da imagem corporal; entre 91 e 110 como moderada distorção; e acima de 110 pontos a classificação é de presença de grave distorção da imagem corporal. ${ }^{21,22}$

\section{Escala de Figuras de Silhuetas (EFS)}

Avalia a percepção do estado atual através de 15 silhuetas de cada gênero. Ela é apresentada em cartões individuais, com variações progressivas na escala de medidas, da figura mais magra (IMC $=12,5 \mathrm{Kg}$ / $\left.\mathrm{m}^{2}\right)$ à mais larga $\left(\mathrm{IMC}=47,5 \mathrm{Kg} / \mathrm{m}^{2}\right)$. Neste teste o sujeito é solicitado a escolher um cartão, dentre os dispostos em série ordenada ascendente, com a silhueta que mais se aproxima da imagem que tem de seu próprio corpo no momento. A presença de distorção da imagem corporal é verificada subtraindo-se o IMC "atual" (escolhido através da escala) do IMC "real" (aferido). ${ }^{12}$

\section{Distorção = IMC "Atual” - IMC Real}

\section{Procedimentos}

A coleta de dados foi realizada em salas cedidas pelas próprias escolas, durante os intervalos das aulas, e os participantes foram divididos em grupos de cinco alunos. Cada sessão durou em média 20 minutos e consistiu na tomada das medidas de peso e estatura, seguida pela aplicação do teste de figuras de silhuetas e auto-aplicação do BSQ após instrução para 
o preenchimento do mesmo. Toda a coleta foi realizada em 2008 pela pesquisadora, previamente treinada para a utilização dos instrumentos.

$\mathrm{O}$ peso foi aferido em balança de plataforma da marca Kratos-Cas (carga máxima de $150 \mathrm{Kg}$ e precisão de $100 \mathrm{~g}$ ), com os alunos posicionados em pé, descalços e trajando roupas leves. A estatura foi aferida em antropômetro vertical, com os alunos também descalços, com os pés paralelos e tornozelos unidos; as nádegas, os ombros e a parte posterior da cabeça encostados em uma parede, estando os braços soltos ao longo do corpo. O aluno permaneceu em pé, ereto, sem encolher ou esticar, olhando para frente. A barra horizontal foi abaixada até repousar no topo da cabeça. A leitura foi efetuada o mais próximo de $0,5 \mathrm{~cm}$.

\section{Análise estatística}

A análise dos resultados foi realizada através da escala de avaliação dos testes e o tratamento estatístico, feito através do software SPSS - versão 8.0, consistiu na aplicação de um teste de normalidade dos dados, seguido de uma análise descritiva dos resultados, além da aplicação de um teste de Correlação de Perason entre os instrumentos e do Teste $t$ de Student para comparação entre os sexos. O nível de significância utilizado foi de $\mathrm{p}<0,05$.

\section{Resultados}

A aplicação do BSQ demonstrou que 14,5\% dos meninos $(n=9)$ e $60,7 \%$ das meninas $(n=34)$ apresentaram algum grau de distorção da imagem de acordo com o BSQ (Figura 1). A média de pontos ( \pm desvio padrão) encontrada pelo questionário foi de 53,8 $( \pm 20,4)$ para os meninos e de $86,2( \pm 34,4)$ para as meninas. Ainda através da Figura 1, observa-se que a EFS demonstrou que no grupo feminino o IMC médio escolhido como "atual" foi de $26,9 \mathrm{Kg} / \mathrm{m}^{2}$, enquanto o IMC médio real foi de $21,5 \mathrm{Kg} / \mathrm{m}^{2} \mathrm{e}$ no grupo masculino, o IMC médio "atual" escolhido foi $23,7 \mathrm{Kg} / \mathrm{m}^{2}$, enquanto a média do IMC real foi de $21,0 \mathrm{Kg} / \mathrm{m}^{2}$. Assim, entre as meninas a distorção da imagem corporal (IMC "Atual" - IMC Real) foi, em média, de $5,47 \mathrm{Kg} / \mathrm{m}^{2}$, enquanto entre os meninos, este valor ficou em torno de $2,74 \mathrm{Kg} / \mathrm{m}^{2}$.

Uma análise estatística mais detalhada demonstrou uma diferença significativa na distorção da imagem corporal entre os sexos pelo BSQ, com maior grau no sexo feminino $[F(1,110)=13,80 ; p<0,001)]$. Entretanto, a EFS não apresentou esta diferença $(\mathrm{p}>0,05)$. (Figura 2).

A aplicação de uma análise de correlação demonstrou uma correlação positiva significativa entre IMC e distorção da imagem pelo BSQ $(r=0,31)$ e pela $\mathrm{EFS}(\mathrm{r}=0,30)$, indicando que quanto maior o IMC, maior é a distorção independente do instrumento utilizado $(\mathrm{p}<0,05)$ (Figura 3), e também entre a distorção da imagem corporal pelo BSQ e pela EFS $(r=0,38 ; p<0,05)$ (Figura 4).

\section{Discussão}

No presente estudo os resultados da aplicação do BSQ demonstraram que $14,5 \%$ dos meninos e
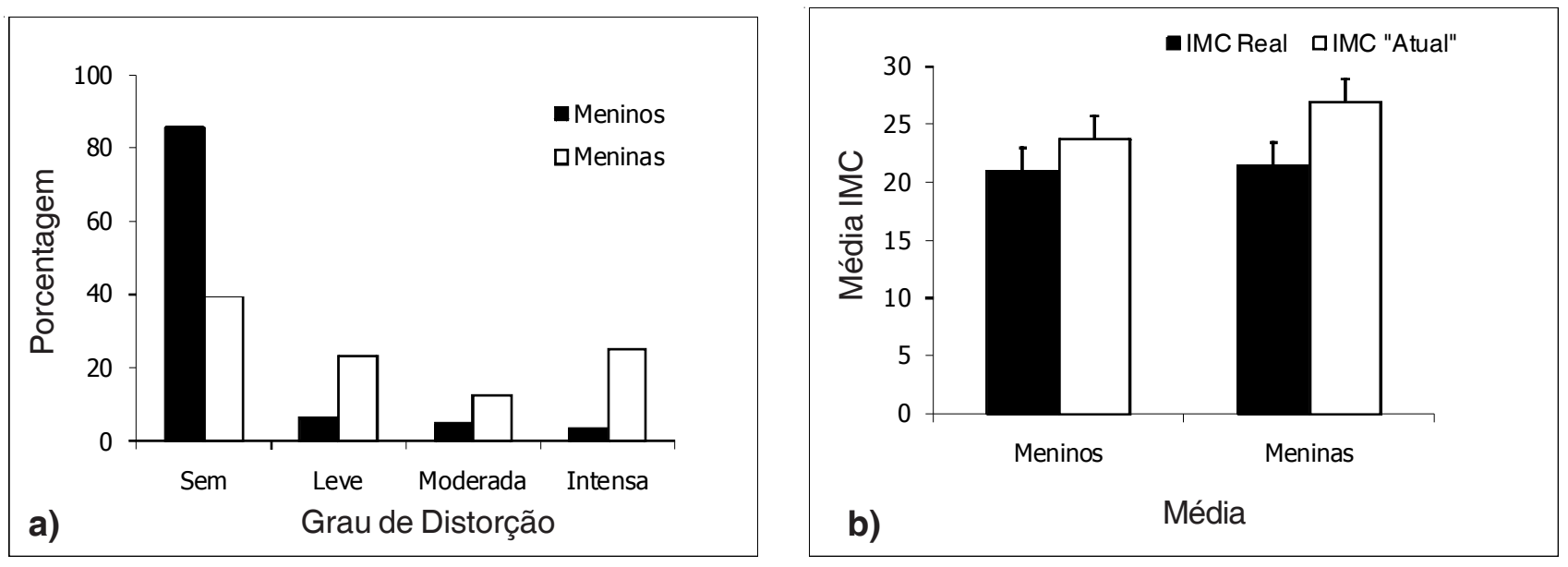

Figura 1. a) Classificação da distorção da imagem corporal dos alunos ( $n=118)$, segundo o BSQ e b) Média ( \pm EPM) do IMC Real e "Atual" dos alunos $(n=118)$, segundo a Escala de Figuras de Silhuetas. 


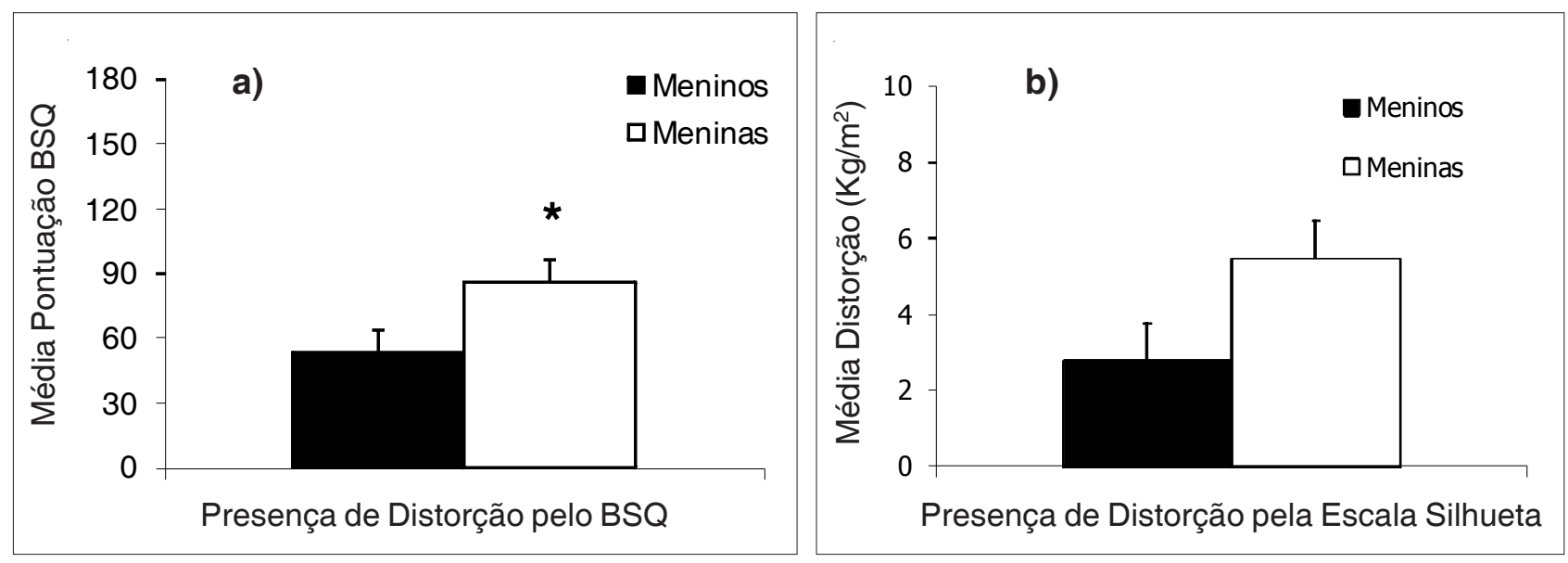

Figura 2. a) Média ( \pm EPM) da distorção da imagem corporal pelo Questionário sobre a Imagem Corporal $(B S Q)$ alunos $(n=118)$. ${ }^{*} p<0,05$ em relação ao grupo dos meninos (Newman-Keuls) e b) Média ( \pm EPM) da distorção da imagem corporal (IMC "Atual" - IMC Real) pela Escala de Figuras de Silhuetas (EFS) nos alunos $(n=118)$. $(p>0,05)$.
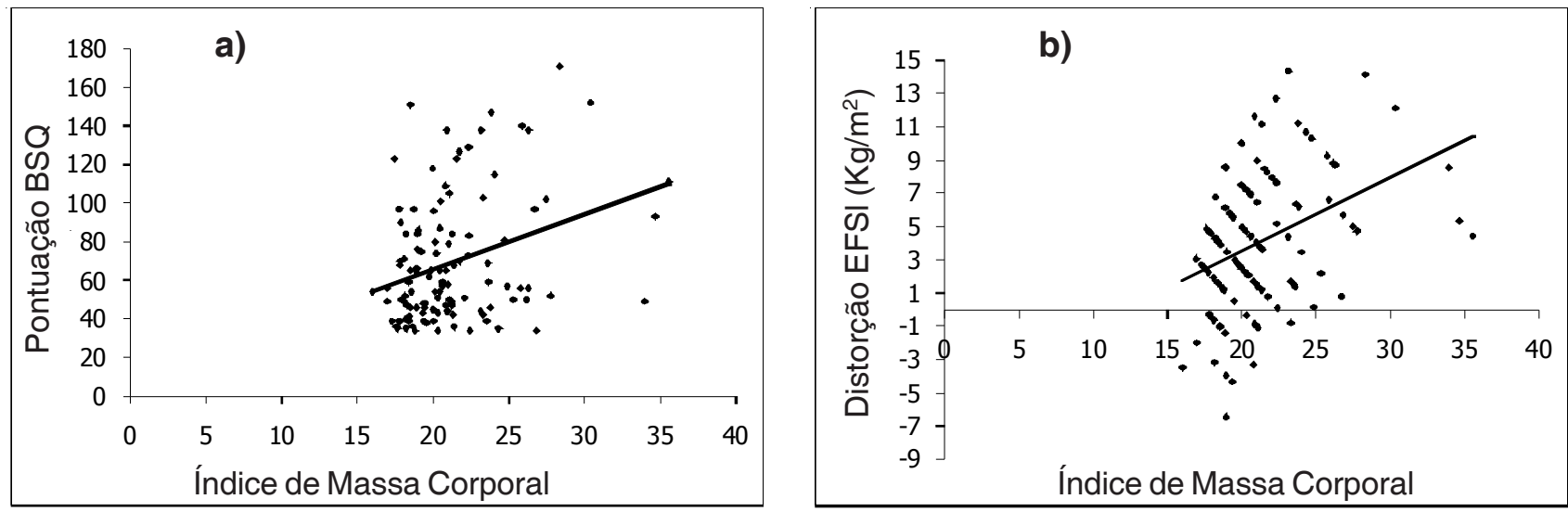

Figura 3. a) Correlação entre o Questionário sobre a Imagem Corporal (BSQ) e o Índice de Massa Corporal $(I M C)$. $(r=0,31)(p<0,05)$ e b) Correlação entre a Escala de Figuras de Silhuetas (EFS) e o Índice de Massa Corporal $(I M C)$. $(r=0,30)(p<0,05)$.

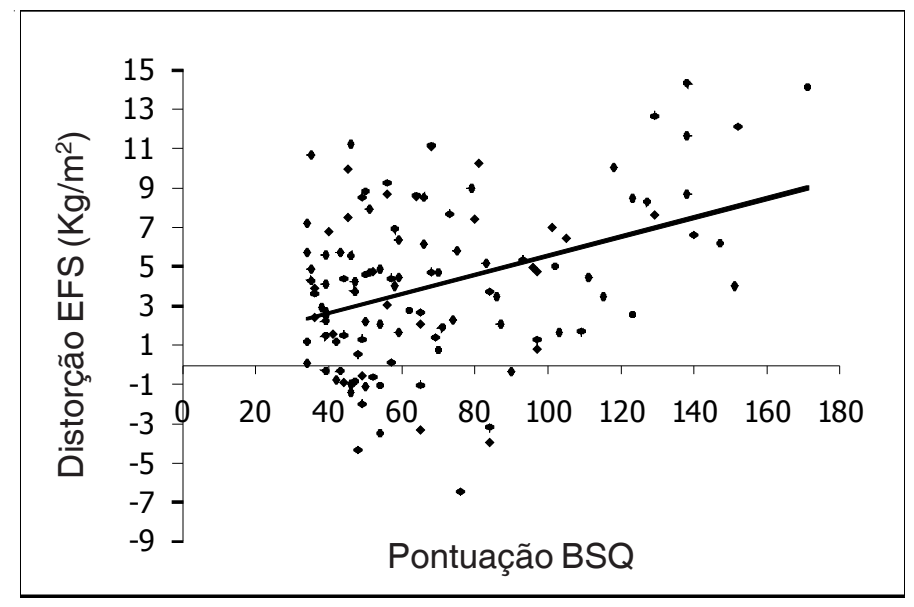

Figura 4. Correlação entre a distorção da imagem corporal medida pelo Questionário sobre a Imagem Corporal (BSQ) e pela Escala de Figuras de Silhuetas (EFS). $(r=0,38)(p<0,05)$. 
60,7\% das meninas apresentaram algum grau de distorção da imagem. Observa-se que, entre as meninas, a distorção intensa foi mais prevalente (25\%), enquanto nos meninos, houve prevalência da distorção leve $(6 \%)$.

Um estudo realizado por Di Pietro ${ }^{8}$ com 164 estudantes universitários demonstrou que a média dos escores do BSQ foi 58,7 $( \pm 25,1)$ para o sexo masculino e $89,7( \pm 31,3)$ para o sexo feminino. Outro estudo, realizado por Vieira et $\mathrm{al}^{23} \mathrm{com} 101$ indivíduos de ambos os sexos, encontrou uma pontuação média para o BSQ de 55,2 $( \pm 22,1)$ para os meninos e 85,5 $( \pm 34,8)$ para as meninas, corroborando dados da presente pesquisa.

Branco et $\mathrm{al}^{24}$ encontraram em seu estudo com 1009 adolescentes entre 14 e 19 anos que 90,3\% dos meninos não apresentavam distorção da imagem por este instrumento, enquanto $41 \%$ das meninas apresentavam algum grau de distorção, sendo a distorção leve a mais prevalente $(22,7 \%)$.

Neste estudo a EFS não apontou uma diferença estatisticamente significante na distorção da imagem corporal entre meninos e meninas, diferentemente dos dados encontrados por Branco et $\mathrm{al}^{24}$, onde a EFS encontrou uma percepção mais comprometida nas meninas ( $\mathrm{n}=1009)$. Uma possível explicação para esta diferença nos resultados pode estar na escala utilizada. Segundo Thompson, ${ }^{25}$ existem mais de 50 escalas que avaliam a percepção da imagem corporal, mas apenas poucas foram validadas.

A exceção da escala de Stunkard et a ${ }^{13}$, que foi validada para adultos brasileiros por Scagliusi et $\mathrm{al},{ }^{26}$ não foram encontrados na literatura trabalhos de validação para o Brasil das escalas de Thompson e Gray ${ }^{14}$ e Madrigal et al, ${ }^{27}$ largamente usadas por pesquisadores brasileiros, enquanto a escala utilizada nesta pesquisa foi adaptada e validada para nossa população, inclusive para adolescentes.

É importante ressaltar que a validade é a questão mais importante a ser proposta com relação a qualquer teste psicológico, uma vez que apresenta uma verificação direta do teste satisfazer sua função. ${ }^{28}$ Não promover estudos nesse sentido é não garantir se o instrumento atendeu às expectativas iniciais. ${ }^{29}$ Além disso, de acordo com Scagliusi et al $^{26}$ uma crítica recorrente ao uso das Escalas de Silhuetas disponíveis na literatura está no pequeno número de figuras apresentadas e consequentemente na restrição da gama de valores que os indivíduos podem selecionar. É possível que um maior número de silhuetas, como é o caso da escala utilizada nesta pesquisa, proporcione mais opções de escolhas, o que pode ter ocasionado a obtenção dos altos índices de distorção encontrados, especialmente no sexo masculino.

Uma vez que, conforme já mencionado, o BSQ foi inicialmente proposto para a avaliação apenas em mulheres, é provável que ele capte melhor a distorção neste sexo. Isto porque a avaliação da percepção da imagem corporal através da Escala de Figuras de Silhuetas não demonstrou diferença entre sexos, indicando que tanto meninos quanto meninas apresentam altos índices de distorção da própria imagem. Esta divergência nos achados poderia ser atribuída a sensibilidades diferentes dos procedimentos utilizados. Uma tentativa de interpretação das diferenças encontradas pode ser feita analisando os instrumentos. O BSQ é um questionário de perguntas diretas mais explícitas que mede cognições e atitudes, enquanto a EFS mede percepção corporal. Ou seja, escalas medindo diferentes componentes da imagem corporal poderiam mostrar resultados diferentes quando utilizadas. ${ }^{30}$

De acordo com Conti et al. ${ }^{31}$ a distorção da percepção corporal não constitui característica particular de adolescentes que desenvolvem algum tipo de TA, uma vez que se torna cada vez mais presente na dinâmica vivencial dos indivíduos dessa faixa etária. Fatores sociais, influências sócio-culturais, pressões da mídia e a busca incessante por um padrão de corpo ideal associado às realizações e felicidade estão entre as causas das alterações da percepção da imagem corporal.

A positividade significativa encontrada na análise de correlação entre os dois instrumentos e entre eles e o IMC demonstra que ambos atuam na detecção do construto estudado. Dessa forma, conforme citado anteriormente, a escolha do instrumento a ser utilizado em cada situação específica deve se pautar na adequação à amostra a ser estudada ${ }^{17}$, uma vez que ambos se assemelham no que diz respeito a seus pressupostos teóricos.

A análise de correlação significativa entre os dois instrumentos obtida no presente estudo é um dado relevante para a literatura da área, visto que não se dispõe de dados anteriores que sugiram que os dois instrumentos possam medir construtos semelhantes.

\section{Conclusão}

Desta forma, com base no exposto, conclui-se que a percepção distorcida da auto-imagem atinge os 
adolescentes, independentemente do sexo. Entretanto, a detecção exata desta distorção só é possível com a escolha correta do instrumento de avaliação. Ambos os instrumentos estudados atuam na detecção de possíveis distorções da imagem corporal, porém, o BSQ conseguiu detectar diferenças devido ao sexo enquanto a EFS parece não ser suficientemente sensível para diferenciar os dois sexos.

\begin{abstract}
Objective: to compare two instruments classically utilized by the literature and indicated to evaluate possible distortions of body image in adolescents and adults of both sexes. Method: the Body Shape Questionnaire (BSQ) and the Silhouette Figure Scale (SFS) was applied in 118 students, aged $16.5 \pm 1.2$ years, 62 boys and 56 girls of 4 public schools. The BSQ measures body shape concerns and the feeling of being "fat"; while the SFS evaluates the actual shape perception and the desirable shape, based on 15 silhouettes of each sex, presented in individual cards, with progressive variations, from the thinner (BMI $\left.=12.5 \mathrm{Kg} / \mathrm{m}^{2}\right)$ to the larger $\left(\mathrm{BMI}=47.5 \mathrm{Kg} / \mathrm{m}^{2}\right)$. Besides that, weight and height were measured for the Body Mass Index (BMI) calculation and posterior comparison with the SFS. The data were submitted to the statistical treatment consisted in a descriptive analysis, a Pearson's correlation test between the instruments and the Student's T-Test for comparison between the sexes. Results: The BSQ showed that $14.5 \%$ of the boys and $60.7 \%$ of the girls presented some level of body image distortion. The SFS on girls showed a "actual" BMI of $26.9 \mathrm{Kg} / \mathrm{m}^{2}$ while the real BMI were $21.5 \mathrm{Kg} / \mathrm{m}^{2}$. The EFS on boys showed a "actual" BMI of $23.7 \mathrm{Kg} / \mathrm{m}^{2}$, while the real BMI were $21.0 \mathrm{Kg} / \mathrm{m}^{2}$, indicating a prevalence of body image distortion in both sexes. The statistical analysis showed a significant positive correlation $(r=0.8)$ between the two instruments, however, the SFS did not presented differences between the sexes $(p=0.93)$, while the $B S Q$ detected a higher level of distortion by the girls $[F(1,110)=13.80 ; p<0.001)]$. Conclusion: the body image distortion was detected by both instruments, however, the BSQ detected differences due to sex, while SFS appears not sufficiently sensible to detect sex differences in adolescents.
\end{abstract}

Keywords: Adolescent. Body Image. Perceptual Distortion. Evaluation Methods.

\section{Referências Bibliográficas}

1. Braggion GF, Matsudo SMM, Matsudo VKR. Consumo alimentar, atividade física e percepção da aparência corporal em adolescentes. Rev Bras Cienc Mov. 2000; 8: 15-21.

2. Nunes MA, Olinto MT, Barros FC, Camey S. Influência da percepção do peso e do índice de massa corporal nos comportamentos alimentares anormais. Rev Bras Psiquiatr. 2001; 23: 21-7.

3. Durkin SJ, Paxton SJ. Predictors of vulnerability to reduce body image satisfaction and psychological wellbeing in response to exposure to idealized female media images in adolescent girls. J Psychosom Res. 2002; 53: 995-1005.

4. Cooper PJ, Taylor MJ, Cooper z, Fairburn CG. The development and validation of the Body Shape Questionnaire. Int J Eat Disord. 1987; 6: 485-94.

5. Freitas S, Gorenstein C, Appolinario JC. Instrumentos para a avaliação dos transtornos alimentares. Rev Bras Psiquiatr. 2002; 24(Supl 3): 34-8.

6. Rosen JC, Jones A, Ramirez E, Waxman S. Body shape questionnaire studies of validity and reliability. Int $\mathrm{J}$ Eat Disord. 1996; 20:315-9.

7. Cordás TA, Castilho S. Imagem corporal nos transtornos alimentares: instrumento de avaliação: Body Shape Questionnaire. Psiquiatr Biol. 1994; 2: 17-21.
8. Di Pietro MC. Validade interna, dimensionalidade e desempenho da escala BSQ - "Body Shape Questionnaire" em uma população de estudantes universitários [Dissertação de Mestrado]. São Paulo: Escola Paulista de Medicina da Universidade Federal de São Paulo; 2002.

9. Gardner RM, Friedman BN, Jackson NA. Methodological concerns when using silhouettes to measure body image. Percept Mot Skills. 1998; 86: 387-95.

10. Gardner RM, Stark K, Jackson NA, Friedman BN. Development and validation of two new scales for assessment of body image. Percept Mot Skills. 1999; 89: 981-93.

11. Madrigal H, Sanches-Villegas A, Martinez-Gonzalez MA, Kearney J, Gibney MJ, Irala J, Martinez JA. Underestimation of body index through perceived body image as compared to self-reported body mass index in the European Union. Public Health. 2000; 114: 468-73.

12. Kakeshita IS. Estudo das relações entre o estado nutricional, a percepção da imagem corporal e o comportamento alimentar em adultos. [Dissertação de Mestrado], Ribeirão Preto: Faculdade de Filosofia, Ciências e Letras - USP; 2004.

13. Stunkard AJ, Sorenson T, Schulsinger F. Use of the Danish adoption register for the study of obesity in thinness. In: Kety S, Rowland LP, Sidman RL, Matthysse SE (Eds.) The genetics of neurological and psychiatric disorders. New York: Raven; 1983. p. 115-20. 
14. Thompson MA, Gray JJ. Development and validation of a new body-image assessment scale. J Personal Assess. 1995; 64:258-69.

15. Kakeshita IS, Almeida SS. Relação entre índice de massa corporal e a percepção da auto-imagem em universitários. Rev Saúde Pública. 2006; 40: 497-504.

16. Leonhard ML, Barry NJ. Body image and obesity: effects of gender and weight on perceptual measures of body image. Addict Behav.1998; 23: 31-4.

17. Thompson JK, Altabe MN, Johnson S, Stormer, S. A factor analysis of multiple measures of body image disturbance: Are we all measuring the same construct? Int J Eat Disord. 1994; 16: 311-5.

18. WHO - World Healt Organization. Physical status: the use and interpretation of anthropometry. Report of a WHO Expert Committee. Geneva, World Health Organization (Technical Report Series), 854, 1995. p.368-9.

19. Barros CASM, Nahra CL. O padrão alimentar anormal em estudantes de Porto Alegre: Levantamento epidemiológico medido pelo EAT-26. Aletheia. 1999; (9): 27-38.

20. CDC - National Center for Chronic Disease Prevention and Health Promotion. Overweight Children and Adolescents: Screen, Assess and Menage. s.d. [citado em 06 Jan 2009] Disponível em: http://www.cdc.gov/nccdphp/dnpa/ growthcharts/training/modules/module3/text/page5f.htm.

21. Assunção SSM, Cordás TA, Araújo LFSB. Atividade física e transtornos alimentares. Rev Psiquiatr Clín (São Paulo). 2002; 29:4-13.

22. 22. Pontieri FM, Lopes PF, Eça VB. Avaliação da presença de fatores de risco para o desenvolvimento de transtornos alimentares em acadêmicos de um curso de educação física. Rev Ciências Biológicas e Saúde [série na internet] 2007 [citado em 3 Dez 2008] Disponível em: http://ww4 unianhanguera.edu.br/programasinst/Revistas/revistas2007/ cienciasbesaude/Avaliacao_da_presenca_de_fatores.pdf
23. Vieira JL, Oliveira LP, Vissoci JRN, Hoshino EF, Fernandes SL. Distúrbios de atitudes alimentares e sua relação com a distorção da auto-imagem corporal em atletas de judô do Estado do Paraná. Rev Educ Fis. /UEM 2006; 17: 177-84.

24. Branco LM, Hilário MOE, Cintra IP. Percepção e satisfação corporal em adolescentes e a relação com seu estado nutricional. Rev Psiquiatr Clín (São Paulo). 2006; 33: 292-6.

25. Thompson JK. The (mis)measurement of body image: Ten strategies to improve assessment for applied and research purposes. Body Image. 2004; 1: 7-14.

26. Scagliusi FB, Alvarenga M, Polacow VO, Cordás TA, Queiroz GKO, Coelho D, Philippi ST, Lancha Jr AH. Concurrent and discriminant validity of the Stunkard's figure rating scale adapted into Portuguese. Appetite. 2006; 47: 77-82.

27. Madrigal-Fritsch $\mathrm{H}$, Irala-Estevez J, Martinez-Gonzalez MA, Kearney J, Gibney M, Martinez-Hernandez JA. Percepción de la imagen corporal como aproximación cualitativa al estado de nutrición. Salud Pública Méx. 1999; 41: 479-86.

28. Pasquali L (Org.) Técnicas de exame psicológico - TEP. Manual. Vol. I: Fundamentos das técnicas psicológicas. São Paulo: Casa do Psicólogo / CFP, 2001

29. Noronha APP, Freitas FA, Ottati F. Parâmetros psicométricos de testes psicológicos de inteligência. Interação Psicol. 2002; 6: 195-201.

30. Cobelo AW. Insatisfação com a imagem corporal e sintomas de transtorno alimentar em mães de adolescentes com transtornos alimentares. [Dissertação de Mestrado], São Paulo: Faculdade de Medicina - USP; 2008.

31. Conti MA, Frutuoso MFP, Gambardella AMD. Excesso de peso e insatisfação corporal em adolescentes. Rev Nutr. 2006; 18: $491-7$. 